\title{
REGULAR FRÉCHET-LIE GROUPS OF INVERTIBLE ELEMENTS IN SOME INVERSE LIMITS OF UNITAL INVOLUTIVE BANACH ALGEBRAS
}

\author{
JEAN MARION AND THIERRY ROBART
}

\begin{abstract}
We consider a wide class of unital involutive topological algebras provided with a $C^{*}$-norm and which are inverse limits of sequences of unital involutive Banach algebras; these algebras are taking a prominent position in noncommutative differential geometry, where they are often called unital smooth algebras. In this paper we prove that the group of invertible elements of such a unital solution smooth algebra and the subgroup of its unitary elements are regular analytic Fréchet-Lie groups of Campbell-Baker-Hausdorff type and fulfill a nice infinite-dimensional version of Lie's second fundamental theorem.
\end{abstract}

\section{INTRODUCTION}

Recent developments in the noncommutative differential geometry originated by A. Connes, particularly in its metric aspects (see [1],[2] and the references therein) and its applications for diverse models in particle physics ([2], [3], [4]), have placed in a prominent position a wide class of unital involutive algebras $\mathbb{A}$ which carry a $C^{*}$-norm and which are inverse limits of suitable sequences of unital involutive Banach algebras; they consitute the noncommutative version of inverse limits of suitable sequences of Banach spaces.

The main goal of this work is to prove that the group GL(A) of invertible elements in $\mathbb{A}$ and the subgroup $\mathcal{U}(\mathbb{A})$ of its unitary elements (playing an important role in the noncommutative geometry picture of Yang-Mills theory $([2],[3]))$ have a canonical structure of regular analytic Fréchet-Lie group of Campbell-Baker-Hausdorff type fulfilling a nice version of Lie's second fundamental theorem.

The present paper is organized as follows:

1991 Mathematics Subject Classification. 17B65, 16A, 58C.

Key words and phrases. Unital involution, ILB-algebra, strong ILB-Lie group, CBHLie group, Lie's second fundamental theorem, Fréchet-Lie group. 
(a) In Section 1 we recall what is the LB-machinery (ILB-chains and strong ILB-Lie groups) initiated by H. Omori ([5] and [6]), as well as their main properties, and we initiate the class of unital involutive ILB-algebras. In the setting of the classical differential geometry a typical example of unital involutive ILB-algebra is given by considering spaces of the type $C^{\infty}(M ; A)$ in which $M$ is a compact smooth manifold and $A$ is a unital $C^{*}$-algebra.

(b) Section 2 is devoted to fundamental examples in the context of noncommutative geometry: starting from a unital involutive algebra $\mathbb{A}$ provided with a $C^{*}$-norm \|\| and using the construction initiated by J. Cuntz in [7], we prove that there exists a unital involutive ILB-algebra $\mathbb{A}^{\prime}$ such that $\mathbb{A} \subseteq \mathbb{A}^{\prime} \subseteq \mathbb{A}_{0}$, where $\mathbb{A}_{0}$ is the $C^{*}$-algebra completion of $\mathbb{A}$ with respect to \|\| , the inclusions being continuous $*$ homomorphisms with dense range.

(c) Section 3 is related to some aspects of differentiability and analyticity on Fréchet spaces according to the ideas of J. Leslie, J. Bochniack, J. Siciak, and some others (see, for example, [8], [9], [6], [10], [5]) and to the notion of generalized Campbell-Baker-Hausdorff Lie group (for shortness: CBH-Lie group) initiated by J. Milnor in [11], which are not necessarily known by nonspecialists in infinite-dimensional analysis and which we shall use in the next sections.

(d) In Section 4 we prove (Theorem 1) that the group GL(A) of invertible elements of any unital involutive ILB-algebra $\mathbb{A}$ has a natural analytic regular Campbell-Baker-Hausdorff Fréchet-Lie group structure with Lie algebra $\mathbb{A}$.

(e) In Section 5 we quote that the group GL(A) fulfills a nice infinitedimensional version of Lie's second fundamental theorem: any closed Lie subalgebra of $\mathbb{A}$ is Lie algebra of a unique connected $\mathrm{CBH}$-Lie group embedded in GL(A) as a Lie subgroup (Theorem 2).

Although this assertion could be most likely deduced from some results of J. Leslie in [10], the type of proof given here is interesting in itself: the proofs generally used for this theorem in the infinite-dimensional context require appropriate versions of the implicit functions theorem and of Frobenius's theorem (see, for example, [1] for the Banach case, and [10] for more general cases with the use of a bornological machinery); in contrast, our proof is only based on some properties of analytic foliations and on the use of the Campbell-Hausdorff formula.

(f) Let $\mathbb{A}$ be any unital involutive topological algebra. The group of its unitary elements

$$
\mathcal{U}(\mathbb{A})=\left\{u \in \operatorname{GL}(\mathbb{A}) \mid u \cdot u^{*}=u^{*} \cdot u=\mathbb{1}\right\}
$$

provided with the induced topology of $\mathbb{A}$ is a topological group. This group plays an important part in the "noncommutative geometry version" of the Yang-Mills theory (see, for example, [2]) and has energy representations 
associated with the image of its "abstract Maurer-Cartan cocycle" by a suitable $K$-cycle over $\mathbb{A}$, even when it is not a Lie group ([12]).

In Section 6 we prove that when $\mathbb{A}$ is a unital involutive ILB-algebra, $\mathcal{U}(\mathbb{A})$ is a regular Fréchet-CBH-Lie subgroup of GL(A) with Lie algebra $\mathcal{K}(\mathbb{A})=\left\{v \in \mathbb{A} \mid v+v^{*}=0\right\}$.

\section{The Class of Initial Involutive IlB-Algebras}

(a) In [5], and especially in [6] to which we refer, H. Omori et al. have indicated what we could call the "the inverse limit Banach-machinery" (to make short: ILB machinery). More precisely (see [6], §6):

Definition 1. (1) A system $\left\{A, A_{k}, k \in \mathbb{N}\right\}$ is called an ILB-chain if $A_{k}$ is a Banach space and $A_{k+1} \subseteq A_{k}$ for any integer $k \geq 0$, the inclusion being continuous and with dense image, and if $A=\cap_{k} A_{k}$ is provided with the inverse limit topology.

(2) A group $\Gamma$ with unit element $e$ is called a strong ILB-Lie group modelled on the ILB-chain $\left\{A, A_{k}, k \in \mathbb{N}\right\}$ if the following conditions are fulfilled for any integer $k \geq 0$ :

(N1): there exists a mapping $\operatorname{Exp}$ from $A$ into $\Gamma$ and for any element $k$ in $\mathbb{N}$ a convex open neighborhood $U_{k}^{0}$ of zero in $A_{k}$ such that Exp is a bijective mapping from $U_{k}^{0} \cap A$ onto an open neighborhood $W_{k}^{0}$ of $e$ in $\Gamma$; we denote by Log the inverse mapping;

(N2): there exists a convex open neighborhood $U_{k}^{1}$ of zero in $A_{k}$ such that

$$
\begin{gathered}
\operatorname{Exp}\left(U_{k}^{1} \cap A\right) \cdot \operatorname{Exp}\left(U_{k}^{1} \cap A\right) \subseteq \operatorname{Exp}\left(U_{k}^{0} \cap A\right) \\
\quad \text { and } \quad \operatorname{Exp}\left(U_{k}^{1} \cap A\right)^{-1} \subseteq \operatorname{Exp}\left(U_{k}^{0} \cap A\right) ;
\end{gathered}
$$

(N3): let $h$ be the mapping from $\left(U_{k}^{1} \cap A\right) \times\left(U_{k}^{1} \cap A\right)$ into $U_{k}^{0} \cap A$ defined by

$$
h(v, w)=\log (\operatorname{Exp}(v) . \operatorname{Exp}(w)) ;
$$

then $h$ can be extended to a continuous mapping (denoted by the same notation) from $U_{k}^{1} \times U_{k}^{1}$ into $U_{k}^{0}$;

(N4): for any $w$ in $U_{k}^{1} \cap A$ the mapping $h_{w}$ defined by $h_{w}(v)=h(v, w)$ is a $C^{\infty}$-mapping from $U_{k}^{1} \cap A$ into $U_{k}^{0} \cap A$;

$(\mathrm{N} 5)$ : $\operatorname{set} \theta(u, v, w)=\left(d h_{w}\right)_{v}(w)$; for any pair $(j, k)$ of elements in $\mathbb{N}$ the mapping $\theta$ can be extended to a $C^{j}$-mapping from $A_{k+j} \times\left(U_{k}^{1} \cap\right.$ $\left.A_{k+j}\right) \times\left(U_{k}^{1} \cap A_{k}\right)$ into $A_{k}$

(N6): the mapping $c$ from $U_{k}^{1} \cap A$ into $U_{k}^{0} \cap A$ defined by $c(v)=$ $\log \left((\operatorname{Exp} v)^{-1}\right)$ can be extended to a continuous mapping from $U_{k}^{1}$ into $U_{k}^{0}$;

(N7): for any element $\gamma$ in $\Gamma$ there exists a neighborhood $V_{k}$ of zero in $A_{k}$ such that

$$
\gamma^{-1} \cdot \operatorname{Exp}\left(V_{k} \cap A\right) \cdot \gamma \subseteq \operatorname{Exp}\left(U_{k}^{0} \cap A\right)
$$


and such that the mapping $v \mapsto \log \left(\gamma^{-1}\right.$. Exp $\left.v \cdot \gamma\right)$ can be extended to a $C^{\infty}$-mapping from $V_{k}$ into $U_{k}^{0}$.

A first important result is that any strong ILB-group is a Fréchet-Lie group ([6], Theorem 6.9).

Let us recall now another important result.

Let $\Gamma$ be a strong ILB-Lie group with unit $\mathbb{1}$ and with Lie algebra $\gamma$; let $C(I ; \gamma)$ be the set of continuous mappings from $I=[0,1]$ into $\gamma$, and let $C^{1, e}(I ; \Gamma)$ be the set of $C^{1}$-mappings $g$ from $I$ into $\Gamma$ such that $g(0)=\mathbb{1}$.

We provide $C(I ; \gamma)$ with the uniform convergence topology and with the algebraic structures pointwise defined from that of $\gamma$ so that it becomes a Fréchet-Lie alebra; likewise, we provide $C^{1, e}(I ; \Gamma)$ with the pointwise group-operations and with the $C^{1}$-uniform convergence topology so that it becomes a FL-Lie group whose Lie algebra consists of the space $C^{1,0}(I ; \gamma)$ of $C^{1}$-mappings $\sigma$ from $I$ into $\gamma$ such that $s(0)=0$ ([6], Lemma 5.2).

It is proved in ([6], Theorems 4.1, 5.1 and 6.9) that

Lemma 1. Any strong ILB-Lie group $\Gamma$ with unit $\mathbb{1}$ and with Lie algebra $\gamma$ is a regular Fréchet-Lie group in the following sense: for any element $s$ in $C(I ; \gamma)$ there exists an element $g_{s}$ in $C^{1, e}(I ; \Gamma)$ satisfying the following equation:

$$
\frac{d g_{s}}{d t}(t)=s(t) \cdot g(t) \quad \text { with } \quad g(0)=\mathbb{1}
$$

Moreover, the assignment $s \mapsto g_{s}$ is a $C^{\infty}$-diffeomorphism from $C(I ; \gamma)$ onto $C^{1, e}(I ; \Gamma)$.

(b) Let us initiate now the ILB-version for unital involutive algebras.

Throughout this paper $\mathbb{K}$ denotes indiscriminately one of the fields of numbers $\mathbb{R}$ or $\mathbb{C}$.

A unital involutive algebra $B$ (always assumed to be associative) over $\mathbb{K}$ being given, as usual we shall denote by $*$ its involution, by $\mathbb{1}$ its unit element, by $\mathrm{GL}(B)$ the group of its invertible elements, and by $\mathcal{U}(B)$ the subgroup of its unitary elements

$$
\mathcal{U}(B)=\left\{u \in \operatorname{GL}(B) \mid u^{*} \cdot u=u \cdot u^{*}=\mathbb{1}\right\} .
$$

By $C^{*}$-norm (resp.: $C^{*}$-seminorm) on $B$ is meant any algebra norm (resp.: algebra seminorm) \|\| on $B$ satisfying $\left\|v^{*} . v\right\|=\|v\|^{2}, v \in B$.

Definition 2. Let $\mathbb{A}$ be a unital involutive topological algebra over $\mathbb{K}$ that we assume to be equipped with a $C^{*}$-norm \|\|$_{0}$. We shall say that $\mathbb{A}$ is a unital involutive ILB-algebra if there exists a sequence $\left\{\left(\mathbb{A}_{k},\|\|_{k}\right)\right\}_{k \in \mathbb{N}}$ of unital involutive Banach algebras satisfying the following properties:

(R1): $\left(\mathbb{A}_{0},\|\|_{0}\right)$ is that $C^{*}$-algebra consisting of the completion of $\mathbb{A}_{k}$ with respect to \|\|$_{0}$; 
(R2): for any integer $k \geq 0, \mathbb{A}_{k+1}$ is a unital involutive subalgebra of $\mathbb{A}$, and the topology of $\mathbb{A}_{k+1}$ given by \|\|$_{k+1}$ is stronger than the one induced by $\left(\mathbb{A}_{k},\|\|_{k}\right)$;

(R3): the system $\left(\mathbb{A}, \mathbb{A}_{k}, k \in \mathbb{N}\right)$ is an ILB-chain that we shall call the ILBA-chain of $\mathbb{A}$.

We note that a unital involutive ILB-algebra $\mathbb{A}$ as a locally convex topological vector space is a Fréchet vector space. The topology of $\mathbb{A}$ being the superior limit of the topologies on $\mathbb{A}$ induced by $\mathbb{A}_{k}$, one easily deduces that the multiplication $m$ from $\mathbb{A} \times \mathbb{A}$ into $\mathbb{A}$ and the involution on $\mathbb{A}$ are continuous and then

Lemma 2. Any unital involutive ILB-algebra is a unital involutive Fréchet algebra.

(c) Of course any unital $C^{*}$-algebra $(\mathbb{A},\|\|)$ is a unital involutive ILBalgebra by taking the ILBA-chain $\left\{\left(\mathbb{A}, \mathbb{A}_{k},\|\|_{k}\right)\right\}_{k \in \mathbb{N}}$ with $\mathbb{A}_{k}=\mathbb{A}$ and \|\|$_{k}=\|\|$ for any $k$ in $\mathbb{N}$.

A typical example of unital involutive ILB-algebra is the set $\mathbb{A}=$ $C^{\infty}(M ; A)$ of smooth mappings from a smooth compact manifold $M$ into a unital $C^{*}$-algebra $A$, provided with the $C^{\infty}$-uniform convergence topology (see, for example, [13]) and with involution and algebraic structure pointwise defined from that of $A$.

In this case, for any $k \geq 1$ the unital involutive Banach algebra $\mathbb{A}_{k}$ is the algebra $C^{k}(M ; A)$ of mappings of class $C^{k}$ from $M$ into $A$ provided with the $C^{k}$-uniform convergence topology, and $\mathbb{A}_{0}$ is the unital $C^{*}$-algebra of continuous mappings from $M$ into $A$ provided with the uniform convergence topology. More generally, one can easily see that the space $C^{\infty}(B)$ of smooth sections of a smooth bundle $B$ over $M$ of unital $C^{*}$-algebras has a natural structure of unital involutive ILB-algebra.

\section{Unital Involutive ILB-Algebras in Noncommutative GEOMETRY}

We want to discuss now a version of unital involutive ILB-algebras in the context of noncommutative geometry in which the notion of smooth algebra takes an important place (see, for example, [14]): in noncommutative geometry, by smooth algebra is meant a pair $\left(\mathbb{A}, \mathbb{A}_{0}\right)$ in which $\mathbb{A}_{0}$ is a unital $C^{*}$-algebra and $\mathbb{A}$ a unital involutive Fréchet dense subalgebra. A unital involutive ILB-algebra $\mathbb{A}$ with its ILBA-chain $\left\{\left(\mathbb{A}, \mathbb{A}_{k},\|\|\right)\right\}_{k \in \mathbb{N}}$ being given, it follows from Lemma 2 that the pair $\left(\mathbb{A}, \mathbb{A}_{0}\right)$ is a smooth algebra.

(a) Let us refer to [7] and the reference therein for detailed construction and results described below.

Let $\mathbb{A}$ be a unital involutive algebra over $\mathbb{K}$, and let $\mathcal{D} \mathbb{A}$ be the universal algebra generated by elements $p_{i}(v), i \in \mathbb{N}, v \in \mathbb{A}$, which are linear in $v$ and 
fulfill the following relations: for any $k$ in $\mathbb{N}$, and any pair $(v, w)$ of elements in $\mathbb{A}$ :

$$
p_{k}(v, w)=\sum_{i+j=k} p_{i}(v) \cdot p_{j}(w) .
$$

$\mathcal{D} \mathbb{A}$ is a $\mathbb{N}$-graded involutive algebra, the degree of $p_{i}(v)$ being the integer $i$, and the involution being given by

$$
p_{i}(v)^{*}=-p_{i}\left(v^{*}\right) ;
$$

moreover, $\mathcal{D} \mathbb{A}$ carries a natural derivation $\delta$ such that

$$
\delta\left(p_{i_{1}}\left(v_{1}\right) \ldots p_{i_{m}}\left(v_{m}\right)\right)=\sum_{k=1}^{k=m}\left(i_{k}+1\right) p_{i_{1}}\left(v_{1}\right) \cdots p_{i_{k}+1}\left(v_{k}\right) \cdots p_{i_{m}}\left(v_{m}\right) .
$$

For any integer $i \geq 0$ we have $p_{i}(v)=\frac{1}{i !} \delta^{i}(v)$ so that, identifying $p_{0}$ with the identity on $\mathbb{A}, \mathcal{D} \mathbb{A}$ is generated by $\mathbb{A}$ and all the $\delta^{i}(\mathbb{A}), i \geq 1$; one obtains then a formal homomorphism $e^{\delta}$ from $\mathbb{A}$ into $\mathcal{D} \mathbb{A}$ by taking

$$
e^{\delta}(v)=\sum_{i=0}^{i=\infty} p_{i}(v)
$$

For any integer $k \geq 0$ let $J_{k} \mathbb{A}$ be the ideal of $\mathcal{D} \mathbb{A}$ generated by all elements with degree $\geq k$; then $e^{\delta}$ leads to an actual homomorphism of algebras from $\mathbb{A}$ into $\mathcal{D} \mathbb{A} / J_{k} \mathbb{A}$ which allows one to consider $\mathbb{A}$ as a subalgebra of $\mathcal{D} \mathbb{A} / J_{k} \mathbb{A}$.

Let us assume moreover that $\mathbb{A}$ is equipped with a $C^{*}$-norm \|\| ; for any integer $k \geq 1$ we provide $\mathcal{D} \mathbb{A} / J_{k} \mathbb{A}$ with the Hausdorff locally convex topological algebra structure by taking the topology given by all algebra seminorms which are bounded by some multiple of \|\| on $\mathbb{A}$ and for which the projection onto the subspace of elements with degree $k$ is bounded.

Let us denote by $\mathbb{A}^{0, k}$ the completion of $e^{\delta}(\mathbb{A})$ in $\mathcal{D} \mathbb{A} / J_{k} \mathbb{A}$, by $\mathbb{A}_{k}^{0}$ the unital involutive topological algebra $\mathbb{A}^{0, k} /\left(\mathbb{A}^{0, k} \cap J_{1} \mathbb{A}\right)$, and by $\mathbb{A}_{0}$ the completion of $\mathbb{A}$ with respect to the $C^{*}$-norm \|\| . We have the dense inclusions of unital involutive Hausdorff locally convex complete topological algebras containing $\mathbb{A}$ :

$$
\cdots \subseteq \mathbb{A}_{k+1}^{0} \subseteq \mathbb{A}_{k}^{0} \subseteq \cdots \subseteq \mathbb{A}_{0}
$$

and $\delta$ extends to a continuous derivation sending $\mathbb{A}_{k+1}^{0}$ into $\mathbb{A}_{k}^{0}, k \in \mathbb{N}$.

(b) We want now to show how the above construction may be connected with unital involutive ILB-algebras. To see this, let us define recursively a norm \|\|$_{(k)}$ on $\mathbb{A}_{k}^{0}$ by \|\|$_{(0)}=\|\|$, and for $k \geq 1$ by

$$
\|a\|_{(k)}=\|a\|_{(k-1)}+\|\delta a\|_{(k-1)}, \quad a \in \mathbb{A}_{k}^{0}
$$


so that for any integer $n \geq 0$ and any $a$ in $\mathbb{A}_{n}^{0}$ one has

$$
\|a\|_{(k)}=\sum_{k=0}^{k=n} \frac{n !}{k !(n-k) !}\left\|\delta^{k} a\right\| .
$$

Let $\mathbb{A}_{k}$ be the completion of $\mathbb{A}_{k}^{0}$ with respect to the norm \|\|$_{(k)}, k \geq 1$, and let $\mathbb{A}^{\prime}=\underset{k \in \mathbb{N}}{\cap} \mathbb{A}_{k}$.

Lemma 3. $\mathbb{A}^{\prime}$ is a unital involutive ILB-algebra with the ILBA-chain $\left(\mathbb{A}^{\prime}, \mathbb{A}_{k}, k \in \mathbb{N}\right)$.

Proof. By definition $\left(\mathbb{A}_{0},\|\|_{0}=\|\|\right)$ is a unital $C^{*}$-algebra, and then a unital Banach algebra; moreover, without loss of generality we may assume that for any pair $(u, v)$ of elements in $\mathbb{A}_{0}$ one has $\|u . v\| \leq\|u\| .\|v\|$; of course, we have also $\left\|u^{*}\right\|=\|u\|$.

Let $u$ and $v$ be in $\mathbb{A}_{1}$. We have

$$
\begin{aligned}
\|u . v\|_{1} & =\|u \cdot v\|+\|\delta(u \cdot v)\|=\|u \cdot v\|+\|\delta u \cdot v+u . \delta v\| \leq \\
& \leq\|u\| \cdot\|v\|+\|\delta u\| \cdot\|v\|+\|u\| \cdot\|\delta v\| \leq \\
& \leq(\|u\|+\|\delta u\|) \cdot(\|v\|+\|\delta v\|) \leq\|u\|_{1} \cdot\|v\|_{1}
\end{aligned}
$$

and $\left\|u^{*}\right\|_{1}=\left\|u^{*}\right\|+\left\|\delta u^{*}\right\|=\left\|u^{*}\right\|+\left\|-(\delta u)^{*}\right\|=\|u\|+\|\delta u\|=\|u\|_{1}$, which proves that $\left(\mathbb{A}_{1},\|\|_{1}\right)$ is a unital involutive Banach algebra; similarly, by induction on $k$, one proves that for any integer $k \geq 0$ and for any pair $(u, v)$ of elements in $\mathbb{A}_{k}$, one has

$$
\|u . v\|_{(k)} \leq\|u\|_{(k)} \cdot\|v\|_{(k)} \text { and }\left\|u^{*}\right\|_{(k)}=\|u\|_{(k)} .
$$

The inclusions $\mathbb{A} \cdots \subseteq \mathbb{A}_{k+1}^{0} \subseteq \mathbb{A}_{k}^{0} \subseteq \cdots \subseteq \mathbb{A}_{0}$ imply the inclusions

$$
\mathbb{A} \cdots \subseteq \mathbb{A}_{k+1} \subseteq \mathbb{A}_{k} \subseteq \cdots \subseteq \mathbb{A}_{0} .
$$

Moreover, the equality $\|a\|_{(k)}=\|a\|_{(k-1)}+\|\delta a\|_{(k-1)}, a \in \mathbb{A}_{k}^{0}$, the continuity of $\delta$, and the density of $\mathbb{A}_{k+1}^{0}$ in $\mathbb{A}_{k}^{0}$ imply that the inclusion of $\mathbb{A}_{k+1}$ in $\mathbb{A}_{k}$ is continuous and that it is with dense range. The assertion is now obvious.

\section{On Differentiable and Analytic Mappings in Fréchet Spaces; Campbell-Baker-Hausdorff Lie Groups}

(a) In infinite-dimensional Hausdorff locally convex vector spaces there are several notions of differentiability and of analyticity that are generally nonequivalent (see, for example, $[6,8,9]$ ), although they coincide with the usual ones in the case of finite-dimensional spaces.

The notion of differentiability used here for Fréchet spaces is that initiated by J. Leslie and which has been taken up in [6], §1, to which we refer: $E$ and $F$ being two Fréchet spaces, and $U$ being some nonempty open subset in $E$, a mapping $f$ from $U$ into $F$ is said to be $C^{0}$ on $U$ if it is continuous 
on $U$. It is called of class $C^{k}$ on $U, k \geq 1$, if it is of class $C^{k-1}$ on $U$, if there exists a continuous mapping $D^{k} f$ from $U \times E^{k}$ into $F$ with the following properties:

- for any $x$ in $U$ the mapping $D^{k} f(x)$ is a symmetric $k$-linear mapping from $E^{k}$ into $F$;

- let $\phi$ be the mapping defined on $E$ by

$$
\phi(v)=f(x+v)-f(x)-(D f(x))(v)-\cdots-\left(D^{k} f(x)\right)(v, \cdots, v) ;
$$

then the mapping from $\mathbb{R} \times E$ into $F$ defined by $R(t, v)=\left\{\begin{array}{l}\frac{\phi(t v)}{t^{k}} \text { if } t \neq 0, \\ 0 \text { if } t=0\end{array}\right.$

is continuous on some neighborhood of $(0,0)$ in $\mathbb{R} \times E$.

Although on infinite-dimensional Banach spaces this notion of differentiability is weaker than the usual definition, it fulfills the chain rule and a nice version of Taylor's expansion.

(b) For the corresponding notion of analyticity we refer to [9]. More precisely, let $E$ and $F$ be two Fréchet spaces and let $U$ be a nonempty subset of $E$; an integer $n \geq 0$ being given, a continuous mapping $p$ from $E$ into $F$ is said to be a continuous homogeneous polynomial with degree $n$ if there exists an $n$-linear mapping $\widehat{p}$ from the Cartesian product $E^{n}$ into $F$ such that

$$
p(v)=\widehat{p}(v, \ldots, v)
$$

for any element $v$ in $E$. We shall denote by $S(E ; F)$ the space of normal series of the form

$$
s=\sum_{n=0}^{n=\infty} p_{n}
$$

where for each integer $n \geq 0 p_{n}$ is a continuous homogeneous polynomial with degree $n$ from $E$ into $F$. In this context a continuous mapping $f$ from $U$ into $F$ is said to be analytic on $U$ if for any element $n$ in $U$ there exists a formal series

$$
s_{x}=\sum_{n=0}^{n=\infty} p_{n, x}
$$

in $S(E, F)$ and an open neighborhood $V_{x}$ of $E$ such that for any $v$ in $V_{x}$

$$
f(x+v)=\sum_{n=0}^{n=\infty} p_{n, x}(v) .
$$

As any Fréchet space is a Banach space, it follows from Theorem 5.2 in [9] that

Lemma 4. If the formal series $s=\sum p_{n}$ in $S(E ; F)$ is convergent on some nonempty open subset $U$ of $E$, the mapping $v \mapsto s(v)=\sum p_{n}(v)$ is analytic on $U$. 
(c) According to J. Milnor's idea, by Campbell-Baker-Hausdorff Lie group (in short, $\mathrm{CBH}-$ Lie group) is meant any analytic Fréchet-Lie group $\Gamma$ with Lie algebra $\gamma$ and unit $\mathbb{1}$ such that the exponential mapping exp from $\gamma$ into $\Gamma$ fulfills the following property: there exists an open neighborhood $U$ of zero in $\gamma$ and an open neighborhood $W$ of $\mathbb{1 1}$ in $\Gamma$ such that the restriction of exp to $U$ is an analytic diffeomorphism from $U$ onto $W$ so that $\left(W, \log =\exp ^{-1}\right)$ is a canonical system of analytic local coordinates for $\Gamma$ near $\mathbb{1}$ (Cf. [11]). As a consequence, the group law near $\mathbb{1}$ is given by the well-known Campbell-Hausdorff formula.

Let $B$ be a unital Banach algebra with Banach algebra norm denoted by \|\| and with unit $\mathbb{1}$, and let $\mathrm{GL}(B)$ be the group of its invertible elements. $B$ has a canonical Banach-Lie algebra structure, the Lie bracket being given by $[v, w]=v \cdot w-w \cdot v,(v, w) \in B \times B$; moreover, without loss of generality, we may assume that there exists a constant $C>0$ such that for any pair $(v, w)$ of elements in $B:\|v \cdot w\| \leq C\|v\| \cdot\|w\|$ and $\|[v, w]\| \leq\|v\| \cdot\|w\|$.

In the next lemma we summarize, without proof, the well-known results about the group GL $(B)$; we refer to [15], Chap. II, $\S 6$ for the proof of these assertions:

Lemma 5. GL $(B)$ has a canonical structure of Banach-Lie group with Lie algebra $B$, its underlying topology being the induced topology of $B$. Let Exp be the mapping from $B$ into $\mathrm{GL}(B)$ defined by

$$
\operatorname{Exp} v \sum_{n \in N} \frac{v^{n}}{n !}, \quad v \in B
$$

Then, Exp is the exponential mapping of $\mathrm{GL}(B)$, and there exist an open neighborhood $U$ of zero in $B$ and an open neighborhood $W$ of $\mathbb{1 1}$ in $\mathrm{GL}(B)$ such that $\operatorname{Exp}$ is an analytic diffeomorphism from $U$ onto $W$ so that $\mathrm{GL}(B)$ is an analytic Lie group; we shall denote by Log the inverse diffeomorphism. As a consequence $(U, \mathrm{Log})$ is a canonical local chart of $\mathrm{GL}(B)$ near $\mathbb{1}$, and the group law in $\mathrm{GL}(B)$ near $\mathbb{1}$ is given by the Campbell-Hausdorff formula

$$
\begin{gathered}
\operatorname{Exp}(v) \cdot \operatorname{Exp}(w)=h(v, w)=v+w+\frac{1}{2}[v, w]+ \\
+\frac{1}{12}([v,[v, w]]-[w,[v, w]])+\cdots
\end{gathered}
$$

We observe in particular that by Lemma $5 \mathrm{GL}(B)$ is a CBH-Lie group for any unital Banach algebra. In contrast, it is known that the Fréchet-Lie group Diff $(M)$ of all diffeomorphisms of a compact smooth manifold is not a CBH-Lie group (see, for example, [11], §9). 


\section{The Lie Group $\operatorname{GL}(\mathbb{A}), \mathbb{A}$ Being a Unital Involutive ILB-ALGEBRA}

(a) Let $\left\{\mathbb{A},\left(\mathbb{A}_{k},\|\|_{k}\right), k \in \mathbb{N}\right\}$ be the ILBA-chain of a unital involute ILB-algebra $\mathbb{A}$. The inverse limit topology on $\mathbb{A}$ is given by all the algebra norms \|\|$_{k}$ restricted to $\mathbb{A}$; taking into account Lemma 5 the group GL(A) appears as the inverse limit of the analytic Banach-Lie groups $\operatorname{GL}\left(\mathbb{A}_{k}\right)$, $k \in \mathbb{N}$, and, moreover, $\operatorname{Exp}_{k}$ denoting the exponential mapping of $\mathbb{A}_{k}$, it is clear that its restriction to $\mathbb{A}$ (still denoted by Exp) maps $\mathbb{A}$ into GL( $\mathbb{A})$. Taking into account this observation one easily deduces that

Lemma 6. The mapping Exp from $\mathbb{A}$ into $\mathrm{GL}(\mathbb{A})$ is a local homeomophism, i.e., restricts to a homeomorphism from some open neighborhood $U$ of zero in $\mathbb{A}$ onto some open neighborhood of $\mathbb{1 1}$ in $\mathrm{GL}(\mathbb{A})$.

For any $u$ in $\operatorname{GL}(\mathbb{A})$ let $L_{u}$ and $R_{u}$ be respectively the left and the right multiplication by $u$ in $\mathbb{A}$ so that $L_{u}(v)=u \cdot v$ and $R_{u}(v)=v \cdot u$ for any $v$ in $\mathbb{A}$; we shall denote by $\operatorname{Ad} u$ the automorphism $L_{u} \circ R_{u^{-1}}$ so that $\operatorname{Ad} u(v)=$ $u \cdot v \cdot u^{-1}$ for any $v$ in $\mathbb{A}$.

Lemma 7. For any $u$ in $\mathrm{GL}(\mathbb{A})$ the mappings $L_{u}, R_{u}$, and $\operatorname{Ad} u$ are analytic $\mathbb{K}$-linear automorphisms of $\mathbb{A}$.

Proof. For any $u$ in $\operatorname{GL}(\mathbb{A}), L_{u}, R_{u}$, and Ad $u$ are clearly algebraic $\mathbb{K}$-linear automorphisms of $\mathbb{A}$; moreover, the equalities $\left(L_{u}\right)^{-1}=L_{u^{-1}},\left(R_{u}\right)^{-1}=$ $R_{u^{-1}}$, and $(\operatorname{Ad} u)^{-1}=\operatorname{Ad} u^{-1}$ show that it suffices to prove the analyticity of the mappings $L_{u}$ and $R_{u}$.

A trivial computation shows that for any $v$ and any $w$ in $\mathbb{A}$ the corresponding Câteaux derivative is given by

$$
\left(L_{u}\right)_{v}^{\prime}(w)=\lim _{t \rightarrow 0} \frac{1}{t}\left(L_{u}(v+t w)-L_{u}(v)\right)=u \cdot w=L_{u}(w) ;
$$

the continuity of the multiplication and the $\mathbb{K}$-linearity of $L_{u}$ imply then that $L_{u}$, as the mapping from $\mathbb{A}$ into itself, is analytic; the proof is similar for $R_{u}$.

Theorem 1. Let $\mathbb{A}$ be a unital involutive ILB-algebra; the group $\mathrm{GL}(\mathbb{A})$ has a canonical structure of regular analytic Fréchet-CBH-Lie group, with Lie algebra $\mathbb{A}$, with exponential mapping Exp defined by

$$
\operatorname{Exp} v=\sum_{n \in \mathbb{N}} \frac{v^{n}}{n !}, \quad v \in \mathbb{A},
$$

and with adjoint representation $\operatorname{Ad}$ of $\mathrm{GL}(\mathbb{A})$ into $\mathbb{A}$ such that for any $u$ in $\mathrm{GL}(\mathbb{A})$

$$
\operatorname{Ad}(u)=\operatorname{Ad} u
$$


Proof. It is divided into four steps.

(1) The notations being as in Lemma 5 , let $W^{\circ}$ be a symmetric open neighhborhood of $\mathbb{1}$ in GL(A) contained in $W$ and fulfilling $W^{\circ} . W^{\circ} \subseteq W$, and let $U^{\circ}$ be the open neighborhood of zero in $A$ defined by $U^{\circ}=\log \left(W^{\circ}\right)$.

For any integer $k$ the family $\left\{v^{n}\right\}_{n \in \mathbb{N}}$ is summable in $\mathbb{A}_{k}$; one easily deduces from Lemma 4 that one can find an open neighborhood $U^{\infty} \subseteq U^{\circ}$ of zero in $\mathbb{A}$ such that for any element $v$ in $U^{\infty}$ the element $\mathbb{1}-v$ is invertible with

$$
(\mathbb{1}-v)^{-1}=\sum_{n \in \mathbb{N}} v^{n},
$$

so that one can find an open neighborhood $W^{\infty} \subseteq W^{\circ}$ of $\mathbb{1}$ in GL(A) such that the "inverse" mapping $u \mapsto u^{-1}$ is analytic in $W^{\infty}$; it siffices to take $W^{\infty}=\operatorname{Exp}\left(U^{\infty}\right)$. By Lemma 6 the mapping Log is a homeomorphism from $W^{\infty}$ onto $U^{\infty}$. It follows from Lemma 7 that for any element $u$ in $\operatorname{GL}(\mathbb{A})$ the set $u . W^{\infty}$ is an open neighborhood of $u$ in $\operatorname{GL}(\mathbb{A})$ and $\log \circ L_{u^{-1}}$ is a homeomorphism from $u . W^{\infty}$ onto $U^{\infty}$. As a consequence GL(A) is an open subset of $\mathbb{A}$.

Moreover, the families

$$
\left\{\frac{v^{n}}{n !}\right\}_{n \in \mathbb{N}} \text { and }\left\{(-1)^{n} \frac{(\mathbb{1}-u)^{n}}{n}\right\}_{n \geq 1}
$$

are summable and the corresponding series which converge: the first to $\operatorname{Exp} v$ in some neighborhood of ero in $\mathbb{A}$, and the second to $\log u$ in some neighborhood of $\mathbb{1}$ in GL(A). It follows then from Lemmas 4 and 5 that there exist an open neighborhood $\Omega$ of zero in $\mathbb{A}$ and an open neighborhood $\Lambda$ of $\mathbb{1}$ in $\mathrm{GL}(\mathbb{A})$ such that the mapping

$$
\operatorname{Exp}: v \mapsto \operatorname{Exp} v=\sum_{n \in \mathbb{N}} \frac{v^{n}}{n !}
$$

is an analytic diffeomorphism from $\Omega$ onto $\Lambda$, the inverse diffeomorphism Log from $L$ onto $W$ being given by

$$
\log u=\sum_{n \geq 1}(-1)^{n} \frac{(\mathbb{1}-u)^{n}}{n} .
$$

At this stage we have proved that GL( $\mathbb{A})$ is an analytic manifold modelled on the Fréchet space $\mathbb{A}$, the underlying topology being the topology induced from $\mathbb{A}$, and that for any element $u$ in $\operatorname{GL}(\mathbb{A})$ the pair

$$
\left(u . \Lambda, \log \circ L_{u^{-1}}\right)
$$

is an analytic chart near $u$. 
(2) To prove that GL $(\mathbb{A})$ is an analytic Lie group modelled on $\mathbb{A}$ it remains to prove the analyticity of the mappings $u-u^{-1}, u \in \mathrm{GL}(\mathbb{A})$, and of the multiplication $m:\left(u, u^{\prime}\right) \mapsto u \cdot u^{\prime}$ on $\operatorname{GL}(\mathbb{A}) \times \operatorname{GL}(\mathbb{A})$.

Using the analyticity of the mappings $u \mapsto u^{-1}$ on some open neighborhood of $\mathbb{1}$ in $\operatorname{GL}(\mathbb{A})$ and the analyticity of the mappings $L_{u^{\prime}}, u^{\prime} \in \mathrm{GL}(\mathbb{A})$, implies clearly the analyticity of the mapping $u \mapsto u^{-1}$ on the whole manifold $\operatorname{GL}(\mathbb{A})$.

Let $u, u^{\prime}$ be elements in $\operatorname{GL}(\mathbb{A})$ and let $v, v^{\prime}$ be elements in $\mathbb{A}$; an easy computation of the Gâteaux derivative $\operatorname{Dm}\left(u, u^{\prime}\right)_{v, v^{\prime}}$ of $m$ at the point $\left(u, u^{\prime}\right)$ following the vector $\left(v, v^{\prime}\right)$ gives

$$
\operatorname{Dm}\left(u, u^{\prime}\right)_{v, v^{\prime}}=u \cdot v^{\prime}+v \cdot u^{\prime}=R_{u^{\prime}}(v)+L_{u}\left(v^{\prime}\right),
$$

from which one deduces that for any $\left(u, u^{\prime}\right)$ in $G L(\mathbb{A}) \times G L(\mathbb{A})$ the linear mapping

$$
\operatorname{Dm}\left(u, u^{\prime}\right):\left(v, v^{\prime}\right) \mapsto \operatorname{Dm}\left(u, u^{\prime}\right)_{v, v^{\prime}}=R_{u^{\prime}}(v)+L_{u}\left(v^{\prime}\right)
$$

is a continuous endomorphism of $\mathbb{A} \times \mathbb{A}$; according to the type of analyticity used here it follows that $m$ is analytic.

(3) According to the general theory of finite or infinite-dimensional manifold the tangent space $T_{1} G L(\mathbb{A})$ of $G L(\mathbb{A})$ at the point $\mathbb{1}$ is the set of equivalent classes of parametrized smooth paths through $\mathbb{1}$ which are defined as follows: let $P(\mathrm{GL}(\mathbb{A}))$ be the set of smooth mappings $p$ from an open neighborhood of zero in $\mathbb{R}$ with values in $\mathrm{GL}(\mathbb{A})$ such that $p(0)=1$; then two elements $p, q$ will be equivalent if the following equality is fulfilled:

$$
\frac{d}{d t}(\log p(t))_{t=0}=\frac{d}{d t}(\log q(t))_{t=0} .
$$

An easy computation shows that $\frac{d}{d t}(\log p(t))_{t=0}=\frac{d p}{d t}(0)$, which belongs to $\mathbb{A}$.

Conversely, for any $v$ in $\mathbb{A}$, let us associate the smooth mapping from $\mathbb{R}$ into $\mathrm{GL}(\mathbb{A})$ defined by

$$
p_{v}(t)=\operatorname{Exp}(t v)
$$

a trivial computation gives $\frac{d}{d t}(\log p(t))_{t=0}=v$ so that $T_{1} \mathrm{GL}(\mathbb{A}) \cong \mathbb{A}$.

It remains to compute the Lie bracket of $\mathbb{A}$ as Lie algebra of $\operatorname{GL}(\mathbb{A})$. To do this, we have to observe first of all that the adjoint representation Ad of $\operatorname{GL}(\mathbb{A})$ into its Lie algebra $\mathbb{A}$ is clearly given by $\operatorname{Ad}(u)=\operatorname{Ad} u=L_{u} \circ R_{u^{-1}}$, $u \in \operatorname{GL}(\mathbb{A})$, with $\operatorname{Ad} u$ analytic by Lemma 7 .

Let $v, w$ be elements in $\mathbb{A}$; according to the general theory of Lie groups the Lie bracket $[v, w]$ must be equal to the image of $w$ by the first derivative 
at the point $u=\mathbb{1}$ in the direction $v$ of the smooth mapping $\eta: u \mapsto$ $\operatorname{Ad} u(w)-w=u \cdot w \cdot u^{-1}-w$ from GL(A) into $\mathbb{A}$; an easy computation gives

$$
\begin{gathered}
{[v, w]=h^{\prime}(\mathbb{1}, v)(w)=\lim _{t \rightarrow 0}\left(\frac{(\mathbb{1}+t v) \cdot w \cdot(\mathbb{1}+t v)^{-1}-w}{t}\right)=} \\
=\lim _{t \rightarrow 0}\left(\frac{1}{t}\left((\mathbb{1}+t v) \cdot w \cdot\left(\sum_{n \geq 0}(-1)^{n} t^{n} v^{n}\right)-w\right)\right)= \\
=\lim _{t \rightarrow 0}\left(\frac{1}{t}\left((\mathbb{1}+t v) \cdot w \cdot\left(\mathbb{1}+t \sum_{n \geq 1}(-1)^{n} t^{n-1} v^{n}\right)-w\right)\right)=v \cdot w-w \cdot v,
\end{gathered}
$$

so that $\mathbb{A}$ is the Lie algebra of $G L(\mathbb{A})$ provided with its canonical Lie bracket.

(4) As for integer $k \geq 0$ the Banach Lie group GL $\left(\mathbb{A}_{k}\right)$ is a CBH-Lie group, and its Campbell-Hausdorff series is convergent (and then is analytic by Lemma 4 ) on some neighborhood of $(0,0)$ in $\mathbb{A}_{k} \times \mathbb{A}_{k}$; one easily deduces that the formal Campbell-Hausdorff series, as element of $S(\mathbb{A} \times \mathbb{A} ; \mathbb{A})$, is convergent on some open neighborhood $\Xi$ of $(0,0)$ in $\mathbb{A} \times \mathbb{A}$.

$\mathbb{A}$ being Fréchet space, it follows that $\mathbb{A} \times \mathbb{A}$ is a Baire space, and then the analyticity of the Campbell-Hausdorff function of GL(A) on $\Xi$ is fulfilled by Lemma 4. As a consequence GL(A) is an analytic Fréchet-CBH-Lie group.

The proof that GL $(\mathbb{A})$ fulfills the properties $(\mathrm{N} 1)-(\mathrm{N} 7)$ described in Definition 1 is now straightforward and easy so that GL(A) is also a strong ILB-Lie group, and then a regular Fréchet-Lie group by Lemma 1. We summarize these properties by saying that GL(A) is a regular analytic Fréchet$\mathrm{CBH}-$ Lie group.

\section{Lie's Second Fundamental Theorem for GL(A)}

(a) For any real or complex finite-dimensional Lie group $G$ with Lie algebra $\mathcal{G}$ we have the following result known as Lie's second fundamental theorem:

"Let $\mathcal{H}$ be a Lie subalgebra of $\mathcal{G}$; then $\mathcal{H}$ is integrable, that is to say, there exists a unique connected Lie group $H$ with Lie algebra $\mathcal{H}$ which can be immersed as Lie subgroup of $G$."

A generalization of this assertion in the infinite-dimensional context forces one, first of all, to consider only closed Lie subalgebras: for closed Lie subalgebras Lie's second fundamental theorem remains true for Banach-Lie groups; we refer to [10] for a detailed discussion of this theorem in the infinite-dimensional case.

Another point is that the property for a mapping $M$ from an infinitedimensional manifold $M$ into another infinite-dimensional manifond $N$ to be an immersion is a very strong property; a weaker property for $\pi$ is to be an embedding, that is to say, a $C^{1}$-mapping on $M$ such that for any $x$ in $M$ the differential $(d \pi)_{x}$ is one-to-one. 
Moreover, even with a stronger hypothesis (by considering only closed Lie subalgebras) and with a weaker result (embedding Lie subgroups instead of immersed Lie subgroups), the proof for an extension of Lie's second theorem meets, for non-Banach Lie groups, with major difficulties or obstructions due to the fact that there is no "nice" theory of differential equations, and a fortiori no "good" Frobenius theorem for the most part of non-Banach Hausdorff locally convex topological vector spaces in spite of some efforts using the bornological machinery (see, for example, [10]).

(b) Theorem 2. Let $\mathbb{A}$ be a unital involutive ILB-algebra provided with its Lie algebra structure of the analytic $C B H-$ Lie group $\mathrm{GL}(\mathbb{A})$. Any closed Lie subalgebra $\mathcal{H}$ of $\mathbb{A}$ is the Lie algebra of a unique connected CBH-Lie group which can be embedded as a Lie subgroup $H$ in $\mathrm{GL}(\mathbb{A})$.

Proof. (1) Let $\mathbb{A}$ be a unital involutive algebra. It follows from Theorem 1 that one can find an open neighborhood $\Lambda$ of $\mathbb{1}$ in the analytic CBH-Lie group GL $(\mathbb{A})$ such that Exp is an analytic diffeomorphism from $\Omega$ onto $\Lambda$ so that $\log$ is an analytic diffeomorphism from $\Lambda$ onto $\Omega$; we consider $\Lambda$ provided with the analytic manifold structure induced by that of GL(A) (or equivalently by that of $\mathbb{A}$ !).

Let $\mathcal{H}$ be a closed Lie subalgebra of the Lie algebra $\mathbb{A}$ so that, as a topological vector subspace of $\mathbb{A}$, it is a Fréchet space. $\Omega \cap \mathcal{H}$ is an open neighborhood of zero on $\mathcal{H}$ that we equip with the induced topology of $\mathcal{H}$. Let us consider now the set

$$
H_{1}=\operatorname{Exp}(\Omega \cap \mathcal{H})
$$

provided with the topology carried from that of $\Omega \cap \mathcal{H}$ by Exp; owing to the analyticity of Exp one easily deduces that $H_{1}$ is an analytic manifold modelled on the Fréchet space $\mathcal{H}$ and regularly embedded in the analytic manifold $\Lambda$. Moreover, the mapping

$$
\left(a, a^{\prime}\right) \mapsto a \cdot a^{\prime}=\operatorname{Exp}(\log a) \cdot \operatorname{Exp}\left(\log a^{\prime}\right)=\operatorname{Exp}\left(h\left(\log a, \log a^{\prime}\right)\right),
$$

where $h$ denotes the analytic mapping defined by the Campbell-Hausdorff formula, is analytic (with respect to the analytic structure of $H_{1} \times H_{1}$ on some nonempty open neighborhood of $(\mathbb{1}, \mathbb{1})$ in $H_{1} \times H_{1}$.

(2) Let $u$ be any element in GL(A); the analyticity of the mappings $L_{u}$ implies that $u . H_{1}$ is an analytic manifold regularly embedded in $u . \Lambda$ and analytically diffeomorphic to $H_{1}$; the mapping $\widehat{u}$ defined for any element $u . a$ in $u . H_{1}$ by $\widehat{u}(u . a)=\log a$ is an analytic diffeomorphism from the open neighborhood $u .\left(\Lambda \cap H_{1}\right)$ onto $\Omega \cap \mathcal{H}$.

Let $\mathcal{L}$ be the union over $\mathrm{GL}(\mathbb{A})$ of all the analytic manifolds $u . \mathcal{H}, u \in$ $\mathrm{GL}(\mathbb{A}) . \mathcal{L}$ is both an analytic vector bundle over GL(A) with fibers of type $\mathcal{H}$, and the invariant distribution of left translation of the Lie subalgebra $\mathcal{H}$. 
Let us prove that $u . H_{1}$, when provided with the chart $\left\{u .\left(\Lambda \cap H_{1}\right) ; \widehat{u}\right\}$, is an integral manifold of $\mathcal{L}$ in a neighborhood of $u$.

Let $a \in u . H_{1} \cap H_{1}$ so that $b=u^{-1}$.a belongs to $H_{1}$; one can find an open neighborhood $V$ of $\mathbb{1}$ in $H_{1}$ such that $b . V$ is an open neighborhood of $b$ in $H_{1}$ and $a . V$ an open neighborhood of $a$ in $H_{1}$. It follows that $u .(b . V)=u .\left(u^{-1} \cdot a \cdot V\right)=a . V$, which is an open subset in $u . H_{1}$ and in $H_{1}$, is then an open neighborhood of $a$ in $u . H_{1} \cap H_{1}$.

(3) We have then proved that the $u . H_{1}, u \in \mathrm{GL}(\mathbb{A})$, are integral manifolds and that the family of open subsets of the $u . H_{1}$ provides a basis for a topology $\Theta$ on GL $(\mathbb{A})$ which is stronger than the induced topology of that of $\mathbb{A}$ (which is the underlying topology of its structure of analytic Lie group).

Let $H$ be the connected component of $\mathbb{1}$ in $\mathrm{GL}(\mathbb{A})$ with respect to $\Theta$. It is clear that $H$ contains $H_{1}$; moreover, the regularity of the embedding of $H_{1}$ in GL(A) implies that $H$ is an analytic manifold. It follows that $H$ is an integral manifold of $\mathcal{L}$ and that $\{u . H\}_{u \in \mathrm{GL}(\mathbb{A})}$ is an analytic foliation of $\mathcal{L}$. As a result it follows that $H$ is a subgroup of $\mathrm{GL}(\mathbb{A})$.

(4) We have now to prove that $H$ is a CBH-Lie group.

Let $\left(u^{\circ}, v^{\circ}\right)$ be an element in $H \times H$, let $w^{\circ}:=u^{\circ} \cdot v^{\circ-1}$, and let $i\left(v^{\circ}\right)$ be the inner automorphism of $\operatorname{GL}(\mathbb{A})$ defined by $i\left(v^{\circ}\right)(u)=v^{\circ} \cdot u \cdot v^{\circ-1}$ which is analytic on $\operatorname{GL}(\mathbb{A})$, since it is the restriction of $\operatorname{Ad} v^{\circ}$ which is analytic on A by Lemma 7 .

The restriction $j\left(v^{\circ}\right)$ of $i\left(v^{\circ}\right)$ to $H$ is an inner automorphism of $H$ and we can find an open neighborhood $Y$ of $\mathbb{1}$ in $H$ such that the set $j\left(v^{\circ}\right)(Y)=Y^{*}$ is contained in $\Lambda \cap H$ so that one easily deduces that $j\left(v^{\circ}\right)$ is analytic (in the sense of the analytic structure of the manifold $H$ ) in the neighborhood $Y^{*}$ of $\mathbb{1}$ in $H$.

Let us point out that for any $(u, v)$ sufficiently near $\left(u^{\circ}, v^{\circ}\right)$ in $H$ so that $u^{\circ-1} \cdot u \cdot v^{-1} \cdot v^{\circ}$ lies in $Y^{*}$, easy computation shows that $u \cdot v^{-1}=w^{\circ} \cdot j\left(v^{\circ}\right)$ $\left(u^{\circ-1} \cdot u \cdot v^{-1} \cdot v^{\circ}\right)$.

Taking into account the analyticity of the left multiplication in $H_{1}$ (and then in $H$ ) and of $j\left(v^{\circ}\right)$ in $Y^{*}$, one deduces that the mapping $\mu$ from $H \times H$ into $H$ defined by $\mu(u, v)=u \cdot v^{-1}$ is analytic in some neighborhood of $\left(u^{\circ}, v^{\circ}\right)$ for any $\left(u^{\circ}, v^{\circ}\right)$ in $H \times H$. It follows that $H$ is an analytic Lie group embedding in GL(A) and its Lie algebra is $\mathcal{H}$. As the exponential mapping of $H$ is clearly the restriction of the exponential mapping of GL(A), one easily deduces that $H$ is a CBH-Lie group.

(5) It remains now to prove the unicity of $H$ up to an isomorphism of Lie groups. Let $H^{\prime}$ be a connected $\mathrm{CBH}$-Lie group embedded in GL(A) and with Lie algebra $\mathcal{H}$; we denote by $s$ the embedding from $H^{\prime}$ into GL(A) and by $s^{*}$ the differential mapping of $s$ at the unit which is then a smooth isomorphism of Lie algebras from the tangent space $T_{1} H^{\prime}$ of $H^{\prime}$ at the unit onto the Lie algebra $\mathcal{H}$.

The unicity of a one-parameter subgroup having the same velocity vector 
at $t=0$ implies that near the unit one has

$$
s(\operatorname{Exp} u)=\operatorname{Exp}\left(s^{*}(u)\right),
$$

which proves that $s$ is a local Lie group isomorphism from $H^{\prime}$ into $H$.

We achieve the proof by observing that, $H$ and $H^{\prime}$ being connected Lie groups, we can find open neighborhoods $W$ and $W^{\prime}$ of the unit in $H$ and $H^{\prime}$, respectively, so that $s$ maps diffeomorphically $W$ onto $W^{\prime}$ such that $H$ is the union of all the W.s. $W=W^{n}$ and $H^{\prime}$ is the union of all the $W^{\prime} . \cdots . W^{\prime}=W^{\prime} n, n \in N$; it follows then that $s\left(H^{\prime}\right)=H$ so that $H^{\prime}$ is actually a $\mathrm{CBH}$-Lie group isomorphic to $H$ via $s$.

\section{The Fréchet-CBH-Lie Group of Unitary Elements of a Unital InVolutive ILB-AlgEBRA}

(a) Let $B$ be any unital topological involutive algebra over $\mathbb{K}$, let $\mathrm{GL}(B)$ be the group of its invertible elements, and let $\mathcal{U}(B):=\left\{u \in B \mid u^{*} \cdot u=\right.$ $\left.u . u^{*}=\mathbb{1}\right\}$ be the subgroup of its unitary elements.

Lemma 8. Provided with the induced topology of $B$ the group $\mathcal{U}(B)$ is a topological group closed in $B$.

Proof. Let $f^{\prime}$ and $f^{\prime \prime}$ be the mappings from $B$ into $B$ respectively defined for any $v$ in $B$ by

$$
f^{\prime}(v)=v \cdot v^{*} \quad \text { and } \quad f^{\prime \prime}(v)=v^{*} \cdot v .
$$

Due to the continuity of the involution and of the multiplication, $f^{\prime}$ and $f^{\prime \prime}$ are continuous mappings; it follows that $\mathcal{U}(B)=f^{\prime-1}(\{\mathbb{1}\}) \cap f^{\prime \prime}-1(\{\mathbb{1}\})$ is closed in $B$. Moreover, the continuity of the multiplication in $B$ implies its continuity in $\mathcal{U}(B)$, and the continuity of the involution on $B$ implies the continuity of $u \mapsto u^{-1}=u^{*}$ on $\mathcal{U}(B)$ so that $\mathcal{U}(B)$ is a topological group.

In the particular case where $B$ is a unital involutive Banach algebra, $\mathrm{GL}(B)$ is a CBH-Banach-Lie group and it is well known that it induces on its subgroup $\mathcal{U}(B)$ a Banach-Lie group structure. Unfortunately, for more general infinite-dimensional Lie groups, due to the lack of local compactness of the underlying space, a closed subgroup of a Lie group is not necessarily a Lie group.

(b) Let $\left(\mathbb{A}, \mathbb{A}_{k} k \in \mathbb{N}\right)$ be the ILBA-chain of a unital involutive ILBalgebra $\mathbb{A}$, let $\theta$ be the involution on $\mathbb{A}$ defined by

$$
\theta(v)=-v^{*}, \quad v \in \mathbb{A},
$$

and let us consider its eigenspaces

$$
\mathcal{K}(\mathbb{A})=\{v \in \mathbb{A} \mid \theta(v)=v\} \quad \text { and } \quad \mathcal{P}(\mathbb{A})=\{v \in \mathbb{A} \mid \theta(v)=-v\} .
$$


Due to the continuity of $\theta, \mathcal{K}(\mathbb{A})$ and $\mathcal{P}(\mathbb{A})$ are closed subspaces of $\mathbb{A}$ and we have the direct sum

$$
\mathbb{A}=\mathcal{K}(\mathbb{A}) \otimes \mathcal{P}(\mathbb{A}) .
$$

Let us consider $\mathbb{A}$ with its canonical structure of Lie algebra of $G L(\mathbb{A})$; for any pair $(v, w)$ of elements of $\mathbb{A}$ one has

$$
[v, w]^{*}=(v \cdot w)^{*}-(w \cdot v)^{*}=w^{*} \cdot v^{*}-v^{*} \cdot w^{*}=-\left[v^{*}, w^{*}\right] .
$$

As an obvious consequence of the above discussion, we have the following result:

\section{Lemma 9.}

(i) $[\mathcal{K}(\mathbb{A}), \mathcal{K}(\mathbb{A})] \subseteq \mathcal{K}(\mathbb{A})$ and $\mathcal{K}(\mathbb{A})$ is a closed Lie subalgebra of $\mathbb{A}$;

(ii) $[\mathcal{K}(\mathbb{A}), \mathcal{P}(\mathbb{A})] \subseteq \mathcal{P}(\mathbb{A})$;

(iii) $[\mathcal{P}(\mathbb{A}), \mathcal{P}(\mathbb{A})] \subseteq \mathcal{K}(\mathbb{A})$.

Remark 1 . Let us observe that $[\mathbb{A}, \mathbb{A}]=\mathbb{A}$; by analogy with what happens for finite-dimensional reductive Lie algebras over $K$, Lemma 5 shows, at least at the algebraic level, a kind of Cartan decomposition of $\mathbb{A}$ with respect to the "Cartan involution" $\theta$.

This statement seems to be all the more justified as we shall see that $\mathcal{K}(\mathbb{A})$ is the Lie algebra of a Lie group which appears as a generalization of compact Lie groups of the type $U(n)$.

Lemma 10. $\mathcal{U}(\mathbb{A})$ is a closed topological subgroup of the $C B H-$ Lie group $\mathrm{GL}(\mathbb{A})$ for the topology induced from that of $\mathbb{A}$; moreover, the exponential mapping Exp maps the Lie algebra $\mathcal{K}(\mathbb{A})$ into $\mathcal{U}(\mathbb{A})$.

Proof. By Lemma 8 the group $\mathcal{U}(A)$ is a subgroup of GL( $\mathbb{A})$ which is closed in $\mathbb{A}$ and then in the Lie group GL($(\mathbb{A})$ which is open in $\mathbb{A}$. Moreover, the continuity of the multiplication in GL(A) implies its continuity in $\mathcal{U}(\mathbb{A})$, and the continuity of the involution implies the continuity of $u \mapsto u^{-1}=u^{*}$ on $\mathcal{U}(\mathbb{A})$, and then $\mathcal{U}(\mathbb{A})$ is a closed topological subsgroup of the analytic Lie group $\mathrm{GL}(\mathbb{A})$.

Let us observe that for any element $v$ in $\mathbb{A}$ we have

$$
(\operatorname{Exp} v)^{*}=\left(\sum_{n \geq 0} \frac{v^{n}}{n !}\right)^{*}=\sum_{n \geq 0} \frac{\left(v^{n}\right)^{*}}{n !}=\sum_{n \geq 0} \frac{\left(v^{*}\right)^{n}}{n !}=\operatorname{Exp}\left(v^{*}\right)
$$

and then for any element $v$ in $\mathcal{K}(\mathbb{A})$

$$
\begin{aligned}
& (\operatorname{Exp} v)^{*} \cdot \operatorname{Exp} v=\operatorname{Exp}(-v) \cdot \operatorname{Exp} v=\mathbb{1}= \\
& =(\operatorname{Exp} v) \cdot \operatorname{Exp}(-v)=(\operatorname{Exp} v) \cdot(\operatorname{Exp} v) *,
\end{aligned}
$$

which proves that $\operatorname{Exp}$ maps $\mathcal{K}(\mathbb{A})$ into $\mathcal{U}(\mathbb{A})$. 
In the case $\operatorname{dim}(\mathbb{A})<\infty$, Lemma 10 allows one to claim that $\mathcal{U}(\mathbb{A})$ is a Lie subgroup of the analytic Lie group GL(A); in the infinite-dimensional case it is not sufficient owing to the lack of local compactness of the underlying space. Fortunately, in our context we have

Theorem 3. Let $\mathbb{A}$ be a unital involutive ILB algebra. The regular Fréchet-CBH-Lie group $\mathrm{GL}(\mathbb{A})$ induces on its closed topological subgroup $\mathcal{U}(\mathbb{A})$ a structure of regular Fréchet-CBH-Lie group with Lie algebra $\mathcal{K}(\mathbb{A})$, the exponential mapping of which is the restriction of $\operatorname{Exp}$ to $\mathcal{K}(\mathbb{A})$ (which we shall also denote by Exp). Moreover, the adjoint representation Ad of $\mathcal{U}(\mathbb{A})$ into $\mathcal{K}(\mathbb{A})$ is given for any $u$ in $\mathcal{U}(\mathbb{A})$ by $\operatorname{Ad} u(v)=$ u.v. $u^{*}, v \in \mathcal{K}(\mathbb{A})$.

Proof. (a) Let $\left(\mathbb{A}, \mathbb{A}_{k} k \in \mathbb{N}\right)$ be the ILBA-chain of the unital involutive ILB-algebra $\mathbb{A}$, and for any $k$ in $\mathbb{N}$ let $\mathcal{U}\left(\mathbb{A}_{k}\right)$ be the group of unitary elements in the unital involutive Banach algebra $\mathbb{A}_{k}$; one easily sees that $\mathcal{U}\left(\mathbb{A}_{k}\right)$ is a CBH-Banach-Lie subgroup of the Banach-Lie group GL $\left(\mathbb{A}_{k}\right)$ with Lie algebra $\mathcal{K}\left(\mathbb{A}_{k}\right)=\left\{v \in \mathbb{A}_{k} \mid v+v^{*}=0\right\}$; moreover, as in the proof of Lemma 10, one proves that the exponential mapping from $\mathcal{K}\left(\mathbb{A}_{k}\right)$ into $\mathcal{U}\left(\mathbb{A}_{k}\right)$ is the restriction to $\mathcal{K}\left(\mathbb{A}_{k}\right)$ of the exponential mapping $\operatorname{Exp}_{k}$ from $\mathbb{A}_{k}$ into $\mathrm{GL}\left(\mathbb{A}_{k}\right)$.

In this context a straightforward check allows one to assert that $\{\mathcal{K}(\mathbb{A})$, $\left.\mathcal{K}\left(\mathbb{A}_{k}\right), k \in \mathbb{N}\right\}$ is an ILB-chain and that

$$
\mathcal{U}(\mathbb{A})=\underset{k \in \mathbb{N}}{\cap} \mathcal{U}\left(\mathbb{A}_{k}\right)
$$

is a strong ILB-Lie group, and then a regular Fréchet-Lie subgroup of GL(A) by Lemma 1.

(b) Let us prove that the tangent space $T_{1}(\mathcal{U}(\mathbb{A}))$ of $\mathcal{U}(\mathbb{A})$ at $\mathbb{1}$, which is necessarily a vector subspace of the tangent space $\mathbb{A}=T_{1}(\mathrm{GL}(\mathbb{A}))$ of $G L(\mathbb{A})$ at $\mathbb{1}$, is exactly $\mathcal{K}(\mathbb{A})$.

According to the general theory of a smooth infinite-dimensional manifold modelled on a Hausdorff locally convex vector space (see, for example, [12], $\S 4)$, an element of $T_{1}(\mathcal{U}(\mathbb{A}))$ is an equivalence class of parametrized smooth mappings $p$ from some open neighborhood of zero in $\mathbb{R}$ taking their values in $\mathcal{U}(\mathbb{A})$ and such that $p(0)=1$. As in the proof of Theorem 1 , one easily proves that it is entirely characterized by the value of $\frac{d p}{d t}(0)$.

As such a path $p$ fulfills the equalities $p(t) \cdot p(t)^{*}=1$ and $\frac{d p^{*}}{d t}(t)=\left(\frac{d p}{d t}(t)\right)^{*}$ for all $t$, one obtains

$$
0=\frac{d}{d t}\left(p \cdot p^{*}\right)(t)=\frac{d p}{d t}(t) p(t)^{*}+p(t) \cdot\left(\frac{d p}{d t}(t)\right)^{*}
$$

for all parameters $t$ so that for $t=0$

$$
\frac{d p}{d t}(0)+\left(\frac{d p}{d t}(0)\right)^{*}=0
$$


which proves that $\frac{d p}{d t}(0)$ lies in $\mathcal{K}(\mathbb{A})$, and then, as $\mathcal{U}(\mathbb{A}) \supseteq \operatorname{Exp}(\mathcal{K}(\mathbb{A}))$, one concludes that $T_{1}(\mathcal{U}(\mathbb{A})) \cong \mathcal{K}(\mathbb{A})$.

The last part of the assertion follows from the fact that the adjoint representation of $\mathcal{U}(\mathbb{A})$ is the restriction to $\mathcal{U}(\mathbb{A})$ of the adjoint restriction of $\operatorname{GL}(\mathbb{A})$ acting on $\mathcal{K}(\mathbb{A})$ and from the fact that for any $u$ in $\mathcal{U}(\mathbb{A})$ and any $v$ in $\mathcal{K}(\mathbb{A}): \operatorname{Ad} u(v)=u \cdot v \cdot u^{-1}=u \cdot v \cdot u^{*}$.

Remark 2. By Lemma $9, \mathcal{K}(\mathbb{A})$ is a closed Lie subalgebra of $\mathbb{A}$; it follows from Theorem 2 that $\mathcal{K}(\mathbb{A})$ is the Lie algebra of a connected $\mathrm{CBH}-$ Lie subgroup $\Gamma$ of GL(A). Due to the unicity of this group one easily deduces that $\Gamma$ is necessarily the connected component $\mathcal{U}_{0}(\mathbb{A})$ of the unit in the Lie group $\mathcal{U}(\mathbb{A})$.

\section{REFERENCES}

1. A. Connes, Géometrie non commutative. InterEditions, Paris, 1990.

2. A. Connes, The metric aspects of noncommutative geometry. Les Houches Lectures, Ecole d'Ete, 1992.

3. A. Connes and J. Lott, Particle models and noncommutative geometry. Nucl. Phys. B 18(1990), 29-47.

4. R. Coquereaux, Non-commutative geometry and theoretical physics. J. Geom. Phys. 6(1989), 425-490.

5. H. Omori, Infinite-dimensional Lie transformation groups. Lect. Notes Math. 247, Springer, New York, 1974.

6. O. Kobayashi, Y. Maeda, H. Omori, and A. Yoshioka, On regular Fréchet-Lie groups IV. Tokyo J. Math. 5(1981), 365-397.

7. J. Cuntz, Representations of quantized differential forms in noncommutative geometry. Preprint, Mathematisches Institut, Univ. Heidelberg, 1992.

8. V. Averbukh and O. Smolyanov, The various definitions of the derivative in linear topological spaces. Russian Math. Surveys 33(1968), 67-113.

9. J. Bochnack and J. Siciak, Analytic functions in topological vector spaces. Studia Math. 39(1971), 77-112.

10. J. Leslie, Some integrable subalgebras of the Lie algebras of infinitedimensional Lie groups. Trans. Amer. Math. Soc. 333(1992), 423-451.

11. J. Milnor, Remarks on infinite-dimensional Lie groups. In: Relativity, Groups and Topology II, Ed. DeWitt and R.Stora, 1007-1059, Elsevier, North Holland, 1984.

12. J. Marion, Energy representations of infinite-dimensional gauge groups in noncommutative geometry. Preprint C.P.T.-C.N.R.S. Marseille $n^{\circ} 2813$ (to appear). 
13. F. Treves, Topological vector spaces, distributions and kernels. Academic Press, New York, 1967.

14. X. Wang, Theorems of Voiculescu Stinespring; extensions of smooth algebras. Preprint College Part, 1992.

15. N. Bourbaki, Groupes et algèbres de Lie. Chap. 2 et 3. Hermann, Paris, 1972.

(Received 01.03.1994)

Authors' addreses:

Jean Marion

C.N.R.C. - Centre de Physique Théorique, Faculté des Sciences de Luminy,

Case 907 F-13288 Marseille Cedex 9

France

Thierry Robart

Centre de Recherches Mathématiques

Université de Montréal

CP 6128-A, Montréal, Québec H3C 3J7

Canada 